\title{
Numerical Model to Study of Contact Force in A Cylindrical Roller Bearing with Technical Mechanical Event Simulation
}

\author{
Laniado-Jacome Edwin \\ Mechanical engineering department, Carlos III University, Madrid, 28911, Spain
}

\begin{abstract}
A study of contact force between rollers and races in a roller bearing with a numerical model for mechanical event simulations bearing is presented in this paper with different load type on shaft. These numerical models provide with the spatial and time distributions of stress and strain values, as well as all the nodal displacements at every time step. The model was developed with the finite element method (FEM) for mechanical event simulations (MES) with the commercial code Algor $^{\mathrm{TM}}$. The model has been validated by verifying that the contact force distributions correspond to those predicted by the analytical model of Harris-Jones.
\end{abstract}

Keywords Mechanical Event Simulation, Finite Element Method, Roller Bearing

\section{Introduction}

There are some studies of the dynamic behavior of bearings, which have very advanced methods of analysis of vibration frequency, but it is difficult to obtain data to generate magnitudes of stress and deformation of the rollers on the races[1-4]. The analysis of experimental models bearing, the method is not sufficient to isolate the signal of movement due to deformation of the load with other signals that are mixed and are known as noise because of this, this work proposes bearing valid model for results of contact[12,13]. This paper proposes a numerical model of cylindrical roller bearing at different speeds of planar rotation in the shaft $(30$, 40,50 and $100 \mathrm{~Hz}$ ) with three degrees of freedom in each node to analyze the contact between rollers with outer race. This is due mainly to the huge computation resources required when many surfaces are to be in contact during a dynamic simulation. Although it varies from one software to another, we can say that the contact between two surfaces $\mathrm{A}$ and $\mathrm{B}$, having NA and NB nodes respectively, involves one contact element for every pair of nodes, which adds up to NA・NB contact elements. To give an example, a 3D numerical model of a 10-ball bearing, with 500 nodes on each ball surface and 500 on each race (inner and outer) surface, requires 5 million contact elements. Note that the velocities concerning rolling bearings demand high time resolution, which leads to extremely large simulations, hav-

* Corresponding author:

elaniado@ing.uc3m.es (Laniado-Jacome Edwin)

Published online at http://journal.sapub.org/jmea

Copyright (C) 2011 Scientific \& Academic Publishing. All Rights Reserved ing many time steps.

To summarize, mechanical event simulations of dynamic phenomena in rolling bearings, like sliding, are, to a certain extent, unapproachable with 3D numerical models and experimental models[2,5,6,7].

In this paper, a $2 \mathrm{D}$ numerical model has been modeled with three degrees of freedom per node for mechanical event simulations of a roller bearing is firstly presented on an analysis of the mechanical behaviour and as data for important contact between the rollers and the outer race. With this model, many simulations have been carried out under the finite element commercial software Algor ${ }^{\mathrm{TM}}$ [8] for different shaft rotation velocities with a radial load applied at its centre. The software provides stress, strains and displacements at each point, for each time step, as well as the contact force on every node involved in the contact between the rolling elements and the races.

The numerical model presented is validated by comparing the load distribution over the outer ring it provides with that according to the counterpart analytical model of JonesHarris.

The formulation of this analytical model and the results it provides concerning the contact force distribution particularized for a rolling bearing identical to the FE/MES modeled. The analysis for the two models for each shaft speed is performed with relative difference on the outer race on the load zone.

The steps followed for this research are as follows.

Numerical model presented is a cylindrical roller bearing with boundary conditions similar to a model of experimental bearing.

Discusses the results of numerical model to find the 
boundaries of contact within the cargo area for each speed of rotation.

This article shows an analytical model of Harris-Jones $[9,10]$ applying the same external and internal conditions have numerical model.

The Harris-Jones model of a roller bearing provides a system of non-linear equations that is resolved with the data analysis program MathCad ${ }^{\mathrm{TM}}[11]$.

An analysis of the relative error between the two models, the numerical model simulated dynamically by means of finite element method in mechanical event simulation and analytic model Harris-Jones.

That is why some authors have performed analytical models with mathematical formulation for analyzing the results of the contact forces on the races[14,15], but the results of these models do not analyze dynamic phenomena such as deformed during early bearing operation, the sliding between mating parts, fatigue, among others.

The way to obtain the contact force distribution from numerical model simulations with the proposed model is shown. Then the corresponding data are compared to those presented, which results in the validation of the proposed model.

\section{Numerical Model of the Cylindrical Roller Bearing}

There are different types of bearing; among the most used in industry are the bearings with balls and cylindrical roller bearings for high load[16]; for this study used the roller cylindrical bearing to study the dynamic behavior and surface contact rollers and races, the main components of this bearing (except for the cage, seals, and other non-loaded components) are basically cylinders whose axes are parallel to each other and to the bearing axis. The dynamics of the system moves on a plane, on the plane normal and the forces between components, as well as the external one, also lie in planes that are parallel to the movement plane: it is a plane stress problem.

The model of the bearing has a shaft with the inner ring, 13 rolling elements (rollers), the outer ring, the cage, and a system of radial elements that gives the prescribed speed to the shaft; adds a new piece is called "motor torque" is applied to control the speed of rotation of the shaft.

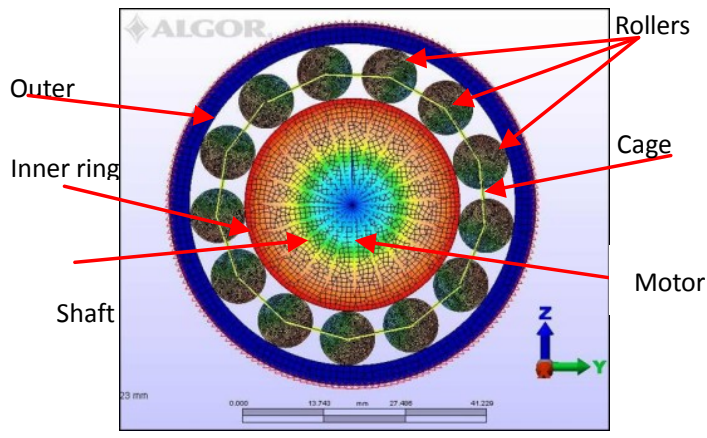

Figure 1. 2D numerical model of the a roller cylindrical bearing
Figure 1 shows the numerical model with 13 roller of cylindrical geometry $(9,20 \mathrm{~mm})$, shaft $(30 \mathrm{~mm})$, the rings (outer $55,10 \mathrm{~mm}$ and inner $36,38 \mathrm{~mm}$ ) and the rollers are meshed into $2 \mathrm{D}$ elements of $10 \mathrm{~mm}$ in length and material properties of common steel (see Table 1), whereas the cage is composed of 13 beam elements articulated at each roller centre. The elements composing the motor torque are beam rigid elements.

Table 1. Material properties of AISI 1006 Steel used for the structural components of the bearing

\begin{tabular}{|c|c|}
\hline Properties & Magnitude \\
\hline Mass density $\left(\mathrm{N} \cdot \mathrm{s}^{2} / \mathrm{mm} / \mathrm{mm}^{3}\right)$ & $7,872 * 10^{-9}$ \\
\hline Young's modulus $\left(\mathrm{N} / \mathrm{mm}^{2}\right)$ & 205000 \\
\hline Poisson's coefficient & 0,29 \\
\hline
\end{tabular}

The model is used in two dimensions to reduce the number of elements of system dynamics. In this research, the total number of elements comes to nearly half a million, from which only about twenty thousand (4\%) are physical ones, while the rest (96\%) are contact elements involved in 26 pairs of surfaces in contact (there are 13 pairs between the roller surfaces and each of the 2 ring surfaces). 1. Title of the paper.

Table 2. Conditions and applied to the model parameters

\begin{tabular}{|c|c|}
\hline Conditions & Magnitude \\
\hline Radial Force & $1000 \mathrm{~N}$ \\
\hline Angular velocity & $30,40,50$ and $100 \mathrm{~Hz}$ \\
\hline Sampling data & 5000 steps per second \\
\hline tolerance distance (nodes of contact) & $0.01 \mathrm{~mm}$ \\
\hline contact friction coefficient & 0.2 \\
\hline contact strength & $10^{5} \mathrm{~N} / \mathrm{mm}^{2}$ \\
\hline
\end{tabular}

Table 2 shows parameters and conditions that define the contact elements employed in our model the tolerance distance (below which the contact element is turned to active) and a constant radial force is applied at the centre of the shaft. All the degrees of freedom of the nodes at the external surface of the outer ring have been restricted. The angular velocity of the shaft is provided by applying prescribed angular motions at the motor torque.

\section{Analytical Model}

The analytical model proposed for a bearing in this research is the Harris-Jones model for a cylindrical roller bearing, this model is generated from the Hertz contact formulation and dynamic balance[17,18]. This model provides a system of non-linear equations composed of one equation for the force balance of the shaft (which is under a radial force applied at its centre), and one more for each of the loaded rollers $(j=1 \ldots z)$, which undergo the contact forces from the inner and outer races as well as the centrifugal force of inertia:

Figure 2 shows the point where the radial force $(\mathrm{Fr})$ is applied and the contact forces in response to applied force on the center shaft. Contact forces are calculated initially on the 
contact points of the rollers on the inner race.

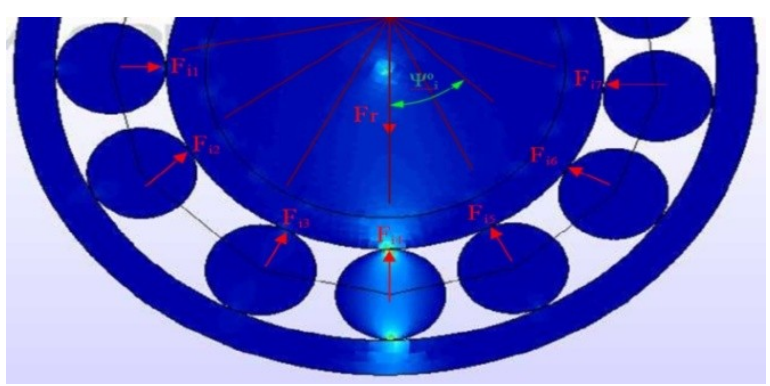

Figure 2. Radial force applied and contact forces on the bearing

$$
\left\{\begin{array}{l}
\frac{F_{r}}{K}-\sum_{j=1}^{j=z}\left(\delta_{i n_{j}}\right)^{10 / 9} \cos \Psi_{j}=0 \\
\left(\delta_{r_{j}}-\delta_{i n_{j}}\right)^{10 / 9}-\left(\delta_{i n_{j}}\right)^{10 / 9}-\frac{F_{c}}{K}=0 ; j=1 \ldots z
\end{array}\right.
$$

$\mathrm{F}_{\mathrm{r}}$ is applied radial force at the centre of the shaft; $\mathrm{K}$ is the force feedback system that causes the material to perform opposition to the radial force, for this model is equivalent contact stiffness between the roller and the races (applying the contact Hertz theory to cylinders, for each contact between a loaded roller and the inner (outer) race.

The normal force is:

$$
\mathrm{F}_{\text {in(out) }}=\mathrm{K}\left(\delta_{\text {in(out })}\right)^{10 / 9}
$$

Contact forces of the rollers on the races are defined with the angle $\left(\Psi_{\mathrm{j}}\right)$; angular position of the $\mathrm{j}_{\mathrm{th}}$ loaded roller with respect to the direction of the applied force (fig.2).

$$
\Delta \Psi^{\mathrm{o}}=360^{\circ} / \mathrm{Z}=27.69^{\circ}
$$

$F_{c}$ is centrifugal force acting on every roller and $n_{i}$ is angular velocity in r.p.m.

$$
\mathrm{F}_{\mathrm{c}}=2.09 \cdot 10^{-7} \mathrm{n}_{\mathrm{i}}^{2}(\mathrm{~N})
$$

$\delta_{\text {in(out)j }}$ is radial deformation corresponding to the contact between the jth roller and the inner (outer) race and $\delta_{\text {irj }}$ is total radial deformation (compression) of the jth roller due to both contacts.

$$
\delta_{\mathrm{rj}}=\delta_{\text {inj }}+\delta_{\text {outj }}=\delta_{\mathrm{r}} \cos \Psi_{\mathrm{j}}
$$

$\delta_{\mathrm{r}}$ is the radial displacement of the inner race center.

The analytical model of Harris-Jones is generated by this system of equations, specifying the model for this study, we have:

Table 3. The symbols employed in Eq. 1

\begin{tabular}{|c|r|}
\hline Symbol & \multicolumn{1}{|c|}{ Magnitude value } \\
\hline $\mathbf{F}_{\mathbf{c}}$ & $2.09 \cdot 10^{-7} \mathrm{n}_{\mathrm{i}}{ }^{2}(\mathrm{~N})$ \\
\hline $\mathbf{Z}$ & 13 roller \\
\hline$\Delta \boldsymbol{\Psi}_{\mathbf{j}}$ & $27.69^{\mathbf{0}}$ \\
\hline $\mathbf{D}$ & $9,2 \mathrm{~mm}$ \\
\hline $\mathbf{F}_{\mathbf{r}}$ & $1000 \mathrm{~N}$ \\
\hline $\mathbf{l}$ & $10 \mathrm{~mm}$ \\
\hline $\mathbf{d}_{\mathbf{m}}$ & $45,74 \mathrm{~mm}$ \\
\hline $\mathbf{K}$ & $608571.25 \mathrm{~N} / \mathrm{mm} 1.11$ \\
\hline
\end{tabular}

Table 3 shows the bearing to apply the system of equations; we get (in S.I. of units):

$0.001643-0.5 \delta_{\mathrm{in} 1}^{1.11}-0.885 \delta_{\mathrm{in} 2}^{1.11}-0.5681 \delta_{\mathrm{in} 3}^{1.11}-0.121 \delta_{\mathrm{in} 4}^{1.11}=0$ (a)

$$
\begin{aligned}
& \left(\delta_{r} \cos 0-\delta_{i n 1}\right)^{1.11}-\delta_{i n 1}^{1.11}-0.00001236=0 \\
& \left(\delta_{r} \cos 27.69-\delta_{i n 2}\right)^{1.11}-\delta_{i n 2}^{1.11}-0.00001236=0 \\
& \left(\delta_{r} \cos 55.38-\delta_{i n 3}\right)^{1.11}-\delta_{i n 3}^{1.11}-0.00001236=0 \\
& \left(\delta_{r} \cos 83.08-\delta_{i n 4}\right)^{1.11}-\delta_{i n 4}^{1.11}-0.00001236=0
\end{aligned}
$$

To solve this nonlinear equation system using the Math$\mathrm{Cad}^{\mathrm{TM}}$ program, entering the solution of the linearized system as the seed for the non-linear one. The error (absolute zero) of this method was less than $10^{-5}$. Finally, the values of the contact forces the outer race exerts on each of the loaded rollers can then be easily obtained from Eq. N:

$$
\mathrm{F}_{\text {outj }}=\mathrm{K}\left(\delta_{\text {in, }}\right)^{10 / 9}+\mathrm{F}_{\mathrm{c}}
$$

With this equation of contact force on the outer race defines the response of the material roller and the outer race to the radial force applied to the center of the shaft and the centrifugal force of the system for each angle $\Psi$.

Table 4. Points of contact deformation of the inner race

\begin{tabular}{|c|c|r|}
\hline Point & Deformation $(\mathbf{m m})$ & \multicolumn{1}{|c|}{ Angle $\boldsymbol{\Psi}^{\mathbf{0}}$} \\
\hline $\boldsymbol{\delta}_{\boldsymbol{i} 1}$ & $2.045^{*} 10^{-3}$ & $0^{\circ}$ \\
\hline $\boldsymbol{\delta}_{\boldsymbol{i} 2}$ & $1.811^{*} 10^{-3}$ & $27.69^{\circ}$ \\
\hline $\boldsymbol{\delta}_{\boldsymbol{i} 3}$ & $1.161^{*} 10^{-3}$ & $55.38^{\circ}$ \\
\hline $\boldsymbol{\delta}_{\boldsymbol{i} 4}$ & $2.443^{*} 10^{-4}$ & $83.08^{\circ}$ \\
\hline
\end{tabular}

Table 4 shows the deformation of the contact points of the roller with the inner race, but not enough to describe the load zone of the bearing model, which is why selecting intermediate points have been contacted by the passage of roller on the race as shown.

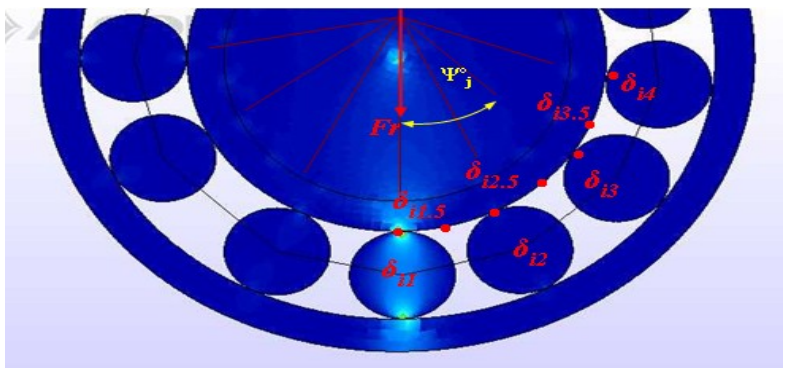

Figure 3. Deformation points on the inner race

Model are added to the deformations due to contact $\delta_{i 1.5}$, $\delta_{\mathrm{i} 2.5}, \delta_{\mathrm{i} 3.5}$, as shown in Figure 3, this creates a load zone with a more accurate geometry. In Figure 4 there is example of load zone for analytical model, the contact force distribution along the outer race is shown for the bearing to be modeled, and for a shaft velocity of 100 revolutions per second.

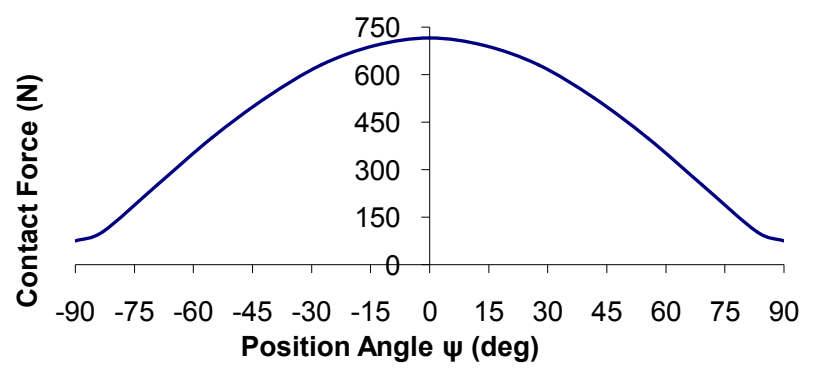

Figure 4. Load zone on outer race to $100 \mathrm{~Hz}$ 
Figure 4 shows the load zone on the outer race of the analytical model from Equation 2.

\section{Numerical Model Contact Force Data}

Data acquisition of the numerical model took into account several factors that met the following dynamic conditions.

Rotation speed of constant shaft.

There is of rolling effect between the rollers and races

Existence of nodes of contact between rollers and races (table 2)

Distance between nodes relatively small to avoid small rebounds.

The mesh of the roller and the inner and outer rings with the contact nodes that are generated are the critical elements involved in the formation of bearing numerical model, which is why you should follow these conditions:

Table 5. Conditions and characteristics of the simulation

\begin{tabular}{|l|c|}
\hline \multicolumn{1}{|c|}{ Conditions } & Magnitude \\
\hline Mesh density & 1400 Joint \\
\hline Angle & $15^{\circ}$ \\
\hline Geometric Factor & 2 \\
\hline refining Factor & 4 \\
\hline Closing Factor & 4 (includes three) \\
\hline Time of calculation & 2400 hours \\
\hline
\end{tabular}

Characteristics and conditions for calculating dynamic numerical model shown in Table 5 are intended to simulate the operation of a bearing with different shaft speeds[5].

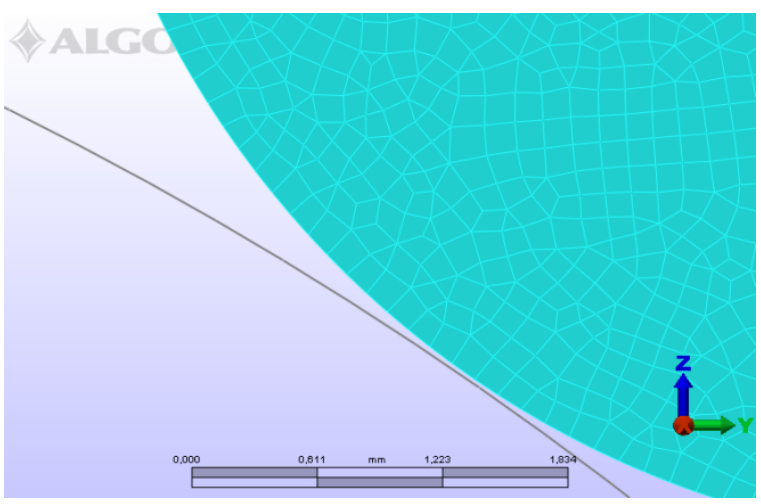

Figure 5. Conditions of meshing for roller

Fig 5 shows the mesh of one of the rollers with the conditions proposed in Table 5 .

Numerical models analyzed at mechanical event simulation has temporal data generated by dynamic phenomenon occurred. Temporary results of contact between the parts (roller with races) of the model is the most important information of this analysis, because are defined in a numerical model with mechanical event simulation, the program calculates the corresponding contact forces for every node, for every time step. Analysis of contact investigations in parts of the bearing support only a small area of deformation between the pieces, in this paper relates an area that is determined by three nodes that deform the roll over by the angle $(\Psi)$ indi-

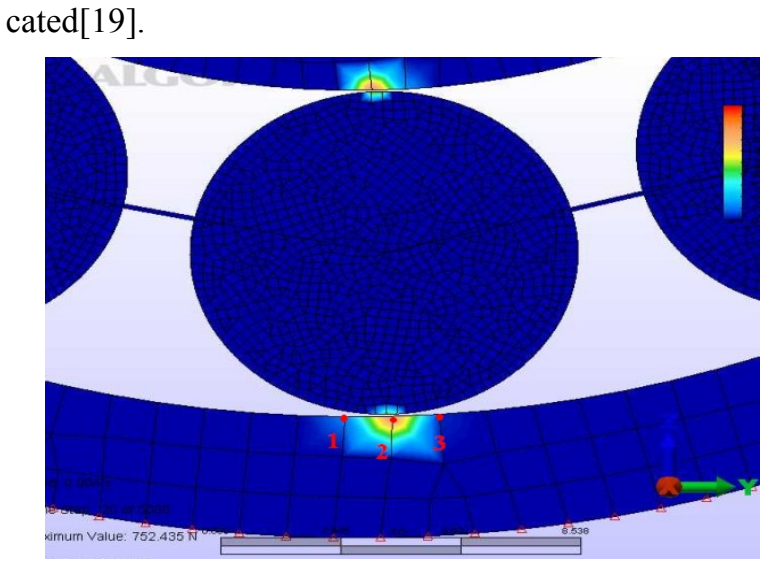

Figure 6. Nodes involved in the contact
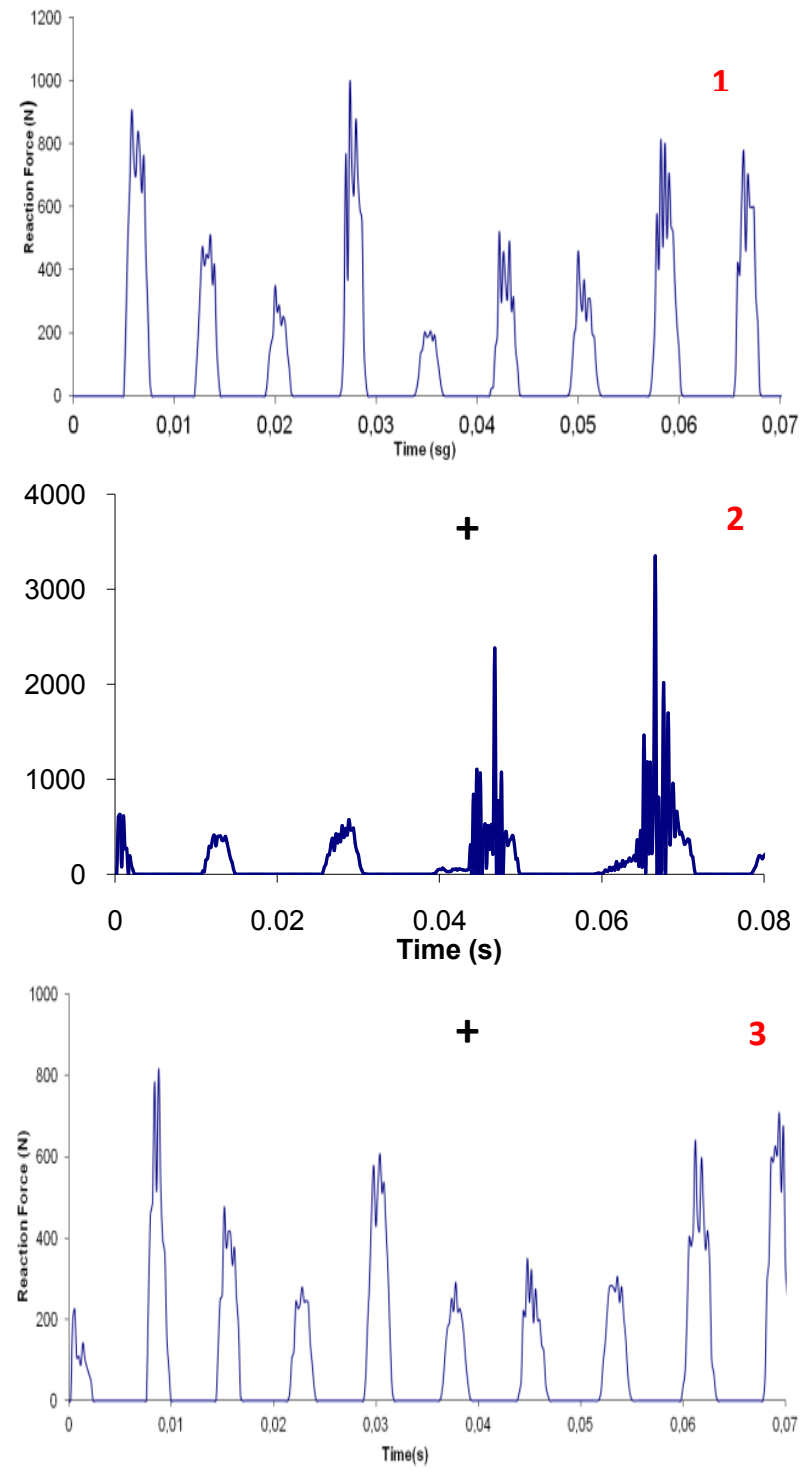

Figure 7. Scheme for the reaction force in the numerical model

Fig 6 shows that due to deformations in the contact between rollers and races, three nodes from the outer race are involved in the contact with a roller in all the simulations (node 1,2, and 3). For this reason, the normal contact force at the position defined by one node in the outer race must be 
obtained by summing up the reaction forces corresponding to this node and its adjacent neighbors.

For this study data were acquired on a temporary basis similar to a signal, for this reason for every angle $(\Psi)$ selected to form the geometry of the loading area were acquired at maximum contact force of the roller to pass through the outer race and has performed a mean (average) with the sum of the maximum reaction force of the three nodes deformed by roller.

Figure 7 shows reaction force has been calculated taking the corresponding media of those maximums for the duration of the simulation for each node that is deformed upon contact with the roller. The sum of the deformations of each node will give the global result of the deflection roller on the outer race for each section. The results of the numerical model will be obtained directly as the reaction force of the rollers on the race under load in Newton. Results were obtained every $13.85^{\circ}$ on the load zone, placing the start of the position angle $\left(\Psi=0^{\circ}\right)$ on the line of force and calling it the center of the zone for all models calculated.

Results were obtained from the $30,40,50$ and $100 \mathrm{~Hz}$ shaft rotation models. The results are temporary with maximum values of force. These values were averaged to obtain the representative value for each angle and the rotation speed for each shaft. These mean values are accompanied by a deviation $(\sigma)$.

$$
\sigma=\sqrt{\frac{\sum(x-\bar{x})^{2}}{(n-1)}}
$$

The deviation shows the interval where the values range for each position angle $\Psi$ (Table 6).

Table 6. Numerical model at $40 \mathrm{~Hz}$, reaction forces on the outer race

\begin{tabular}{|c|c|c|}
\hline Angle ( $\Psi$ ) & Reaction Force & Deviation \\
\hline-90.00 & 55.00 & 4.56 \\
\hline-83.08 & 55.08 & 14.12 \\
\hline-69.24 & 197.93 & 15.33 \\
\hline-55.38 & 409.09 & 28.25 \\
\hline-41.54 & 528.75 & 44.02 \\
\hline-27.69 & 627.79 & 39.02 \\
\hline-13.85 & 687.00 & 38.49 \\
\hline 0 & 737.59 & 32.99 \\
\hline 13.85 & 704.88 & 34.67 \\
\hline 27.69 & 521.16 & 26.29 \\
\hline 41.54 & 471.88 & 22.17 \\
\hline 55.38 & 374.32 & 15.13 \\
\hline 69.24 & 222.52 & 5.85 \\
\hline 83.08 & 107.43 & 6.57 \\
\hline 90.00 & 75.00 & 4.12 \\
\hline
\end{tabular}

The maximum value of the deviation does not exceed $8 \%$ of the value of the reaction force in this research, shown as an example in Table 4 for each angle $(\Psi)$.

Uniting the average values of the force deviation gives the geometry of the load zone of the bearing numerical model at 30, 40, 50 and $100 \mathrm{~Hz}$ rotational speed of the shaft.

The average values of reaction forces shown in Fig 8 represent the passage of the roller on each position angle of $13.85^{\circ}$ and are joined by a trend line of exponent 4 to form the geometry of the load zone for each speed of rotation. In
Fig. 8 shows that the higher the shaft speed, the deviation decreases and the load zones improve its geometry being better defined.
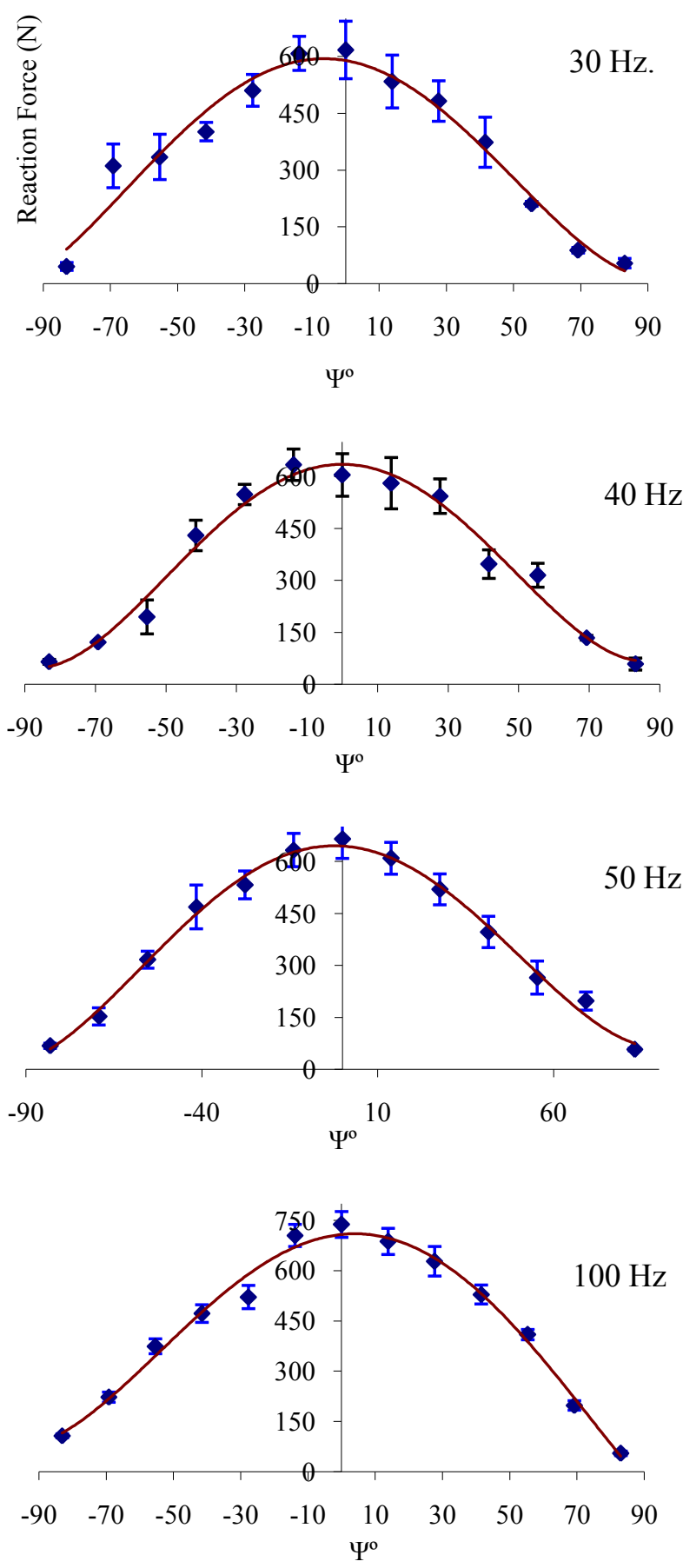

Figure 8. Reaction force with deviation on load zone

\section{Validation with Relative Difference between Models}

Is validated by comparison of two theoretical models, is not defined experimental model because of the difficulty of acquiring data of contact between the rolling elements and 
the rings of a bearing. The analytical model shown in this study of the dynamic equilibrium equations of Harris and Jones proposed to determine the reaction force within the load zone for this study assumes that forces the load zone are low magnitude.

The validation of the numerical model of a cylindrical roller bearing for this research was conducted by comparison with the analytical model of Harris - Jones with similar features and equal boundary conditions. The validation was performed by comparing the reaction force exerted by way of the rollers on the outer race in the load zone for both models.

For a real validation have been described the geometry of the load zone for each model, the description of the load zone will serve to better demonstrate the dynamic behavior of the numerical model compared with the analytical model.
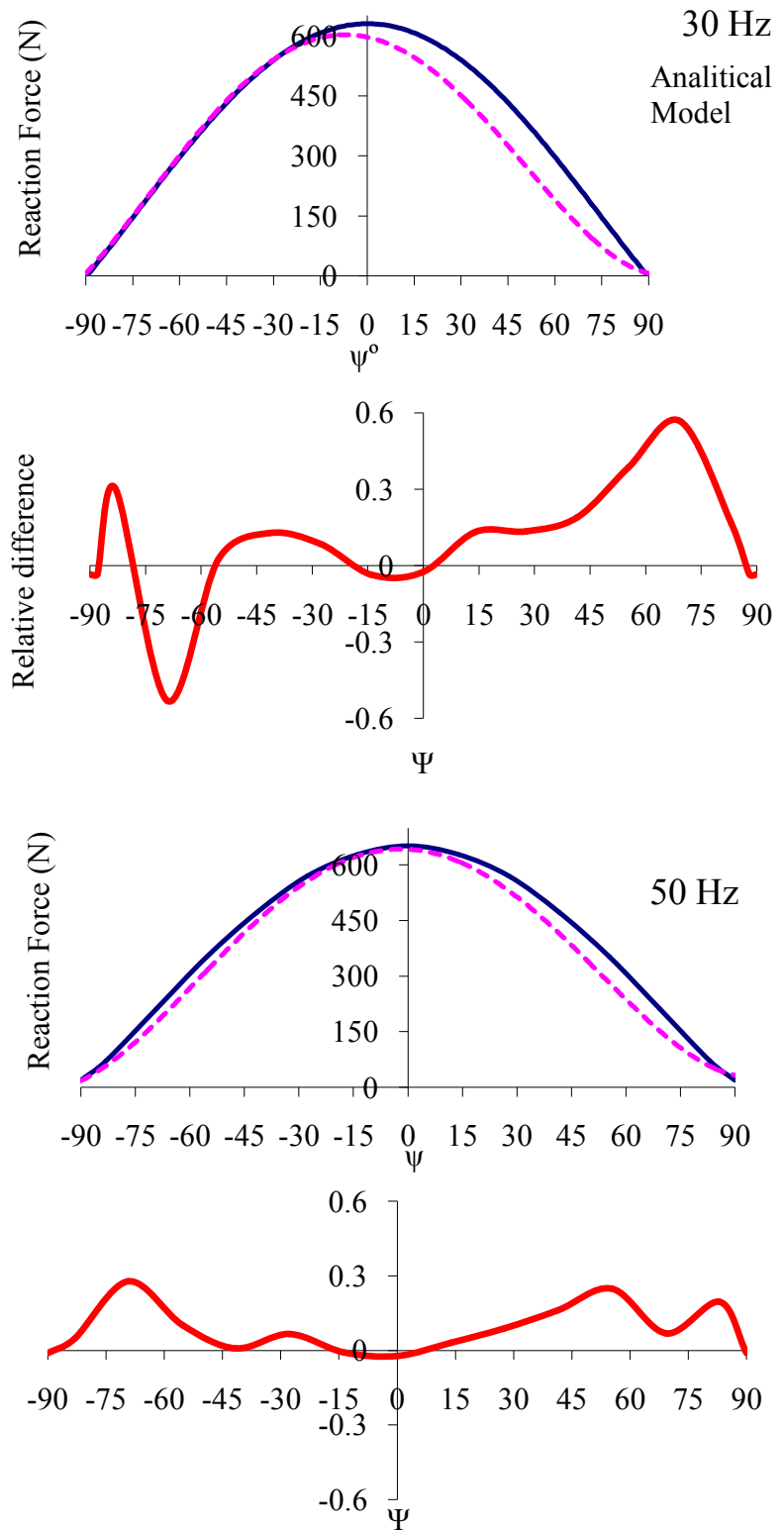

Figure 9. Load zones of analytical and numerical 30, 40, 50 and $100 \mathrm{~Hz}$ models
Figure 9 shows the load zones with the relative difference or potential error that occurs throughout the length of the outer ring. The analysis shows that in the area of maximum contact in the center of the load zone, the differences between the two models are small, less than 0.1 in magnitude, critical area between the angles $(\Psi)-30^{\circ}$ to $30^{\circ}$, important to know the angular range because it may more accurately predict evolution of a localized defect.

The chaos generated by the dynamics of the numerical model are shown in graphs that show the relative difference, This dynamic phenomenon is not shown in the analytical model analysis, because the mathematical equations of equilibrium used in the analysis of the analytical model does not predict random events such as shock, misalignment, etc. due to the drop shaft functioning during early bearing.

Load zones are symmetric analytical models for the position of the angle in the negative direction and a positive difference in the load zone of the numerical model has no symmetry. The differences in the values of the numerical model with respect to the analytical model showed in Fig 6 show no major differences in the magnitude of force along the angle $\Psi$.

\section{Conclusions}

Conditions and characteristics applied to the numerical model of a cylindrical roller bearing are similar to the experimental design, but although the signals and data acquired from the analysis of numerical model are as close to a real model known.

Numerical model of a cylindrical roller bearing has been validated through the optimum analytical model proposed by Harris-Jones without major differences. Presented differences are because the dynamic numerical model presents phenomena that are manifest in the load zone is not symmetrical.

The numerical model presented phenomena of an experimental model; these phenomena are due to the dynamics of the system, where you can explore different events as chaotic behavior of the rolling elements, falls, blows, slipping between the races and rollers, among others.

Numerical model generates the geometry of the load zones adapted to the dynamics of the bearing elements in contact with geometry it may predict behavior, failure, location of critical areas among others.

\section{REFERENCES}

[1] S. Braun, "Mechanical signature analysis of sonic bearing vibrations," IEEE Transactional on Sonic and Ultrasonics, vol. 27, pp. 317-328, 1980

[2] Z. Kiral and H. Karagülle, "Vibration analysis of rolling element bearings with various defects under the action of an 
unbalanced force," Mechanical Systems and Signal Processing, 2005

[3] R. Rubini and U. Meneghetti, "Application of the Envelope and Wavelet Transform Analyses for the Diagnosis of Incipient Faults in Ball Bearings," Mechanical Systems and Signal Processing, vol. 15, pp. 287-302, 2001

[4] S. Wang, W. Huang and Z. K. Zhu, "Transient modeling and parameter identification based on wavelet and correlation filtering for rotating machine fault diagnosis," Mechanical Systems and Signal Processing, vol. 25, pp. 1299-1320, 2011

[5] Zienkiewicz, O.C. and Cheung, Y.K., The Finite Element Method in Structural and Continuum Mechanics. New York: MacGraw-Hill, 1967

[6] Sawalhi, N. and Randall, R.B., "Simulating gear and bearing interactions in the presence of faults Part I. The combined gear bearing dynamic model and teh simulation of localised bearing faults," Tribology International, vol. 22, pp. 1924-1951, 2008

[7] I. A. Zverev, I. -. Eun, W. J. Chung and C. M. Lee, "Simulation of Spindle Units Running on Rolling Bearings," International Journal of Advanced Manufacturing Technology, vol. 21, pp. 889-895, 2003

[8] ALGOR ${ }^{\mathrm{TM}}$. (2008, License number DE23692. I+D+I. professional multiphysics. MES. 19

[9] T. A. Harris, Rolling Bearing Analysis. New York, U.S.A.: 1991

[10] A. Jones, "A general theory for elastically constrained ball and Roller bearing under arbitrary load and Speedy conditions." vol. 105, pp. 591-595, 1960
[11] MATHCAD ${ }^{\mathrm{TM}}$., "License Universidad Carlos III de Madrid. PTC," vol. 14, 2005

[12] S. Braun, Discover Signal Processing. An Interactive Guide for Engineer. 2008

[13] B. K. Bae and K. J. Kim, "A Hilbert transform approach in source identification via multiple-input, single-output modelling for correlated inputs," Mechanical Systems and Signal Processing, vol. 12, pp. 501-513, 1998

[14] S. Harsha, "Nonlinear dynamic analysis of an unbalanced rotor supported by roller bearing. Chaos, Solitons and Fractal," Chaos, Solitons and Fractal, vol. 26, pp. 47-66, 2005

[15] N. Tandon and A. Choundhury, "An analytical model for the prediction of the vibration response of rolling element bearings due to localized defect," Journal of Sound and Vibration (1997) 205 83), 275-292., pp. 275-292, 1997

[16] S. Orhan, N. Aktürk and V. Celik, "Vibration monitoring for defect diagnosis of rolling element bearings as a predictive maintenance tool," Comprehensive Case Studies NDT\&E International, vol. 39, pp. 293-298, 2006

[17] H. Hertz, "Über die Berührung fester elastischer Körper " J. Reine Angew Math, vol. 92, pp. 156-71., 1882

[18] Kang, Y., Shen, P., Huang, C., "A modification of the Jones-Harris method for deep-groove ball bearings," vol. 39, pp. 1413-1420, 2006

[19] P. J. L. Fernandes, "Contact fatigue in rolling-element bearings," Engineering Failure Analysis, vol. 4, pp. 155-160, 1997 